\title{
Association of HOTAIR polymorphisms rs4759314 and rs920778 with cancer susceptibility on the basis of ethnicity and cancer type
}

\author{
Qichao Qi ${ }^{1, *}$, Jiwei Wang ${ }^{1, *}$, Bin Huang ${ }^{1}$, Anjing Chen ${ }^{1}$, Gang Li $^{1}$, Xingang Li ${ }^{1}$, Jian \\ Wang $^{1,2}$ \\ ${ }^{1}$ Department of Neurosurgery, Qilu Hospital of Shandong University and Brain Science Research Institute, Shandong \\ University, Jinan, 250012, China \\ ${ }^{2}$ Department of Biomedicine, University of Bergen, Bergen, 5009, Norway \\ *These authors have contributed equally to this work \\ Correspondence to: Xingang Li, email: lixg@sdu.edu.cn \\ Jian Wang, email: jian.wang@biomed.vib.no
}

Keywords: cancer, genetic susceptibility, HOTAIR, polymorphism

Received: January 26, $2016 \quad$ Accepted: May 02, $2016 \quad$ Published: May 26, 2016

\section{ABSTRACT}

Polymorphisms in the HOX transcript antisense intergenic RNA (HOTAIR) have been recently associated with susceptibility to different cancers. Here, a meta-analysis was performed to derive a more precise estimation of the involvement of HOTAIR polymorphisms in cancer development. Data from cases $(n=7,772)$ and controls $(n$ $=9,075)$ were extracted from eligible studies $(n=10)$ identified in a comprehensive literature search conducted in PubMed, Embase, and the Web of Science databases through January 20, 2016. Overall, association between polymorphism rs920778 and increased cancer risk was significant in allele contrast (odds ratio $(O R)=1.239$, $95 \%$ confidence interval $(\mathrm{CI})=1.032-1.487)$ and recessive models $(\mathrm{OR}=1.614$, $95 \% \mathrm{CI}=1.082-2.406$ ). In subgroup analysis based on ethnicity, a significant association between polymorphism rs920778 and cancer susceptibility was observed in Asians under all models, but was most compelling under recessive (OR $=\mathbf{2 . 1 2 8}$, $95 \% \mathrm{CI}=1.417-3.197)$ and homozygous models $(\mathrm{OR}=2.764,95 \% \mathrm{CI}=2.221-$ 3.440). Subgroup analysis by cancer type revealed a significant association between polymorphism rs4759314 and susceptibility to gastric cancer in allele contrast (OR $=1.262,95 \% \mathrm{CI}=1.073-1.486)$, dominant $(\mathrm{OR}=1.280,95 \% \mathrm{CI}=1.060-1.547)$, and heterozygous models $(O R=1.288,95 \% \mathrm{CI}=1.057-1.570)$. In conclusion, the results indicated that HOTAIR polymorphism rs920778 was more generally associated with cancer risk, particularly in Asians, while rs4759314 was a risk factor for gastric cancer.

\section{INTRODUCTION}

Long non-coding RNAs (lncRNAs) are defined as transcribed RNA molecules that are longer than 200 nucleotides and not translated into proteins [1]. Although their function was initially unclear, lncRNAs are now known to have critical roles in the regulation of gene expression through transcription, processing of small RNAs, and epigenetic modification as well as other regulatory functions $[2,3]$. As key components in gene regulatory complexes, IncRNAs contribute to the activation or inhibition of expression of genes involved in diverse normal cellular processes, such as proliferation and apoptosis. Many of these same processes are corrupted in cancer, and thus deregulated lncRNA expression has been linked to development of the disease [3-6].

Increasing evidence indicates that one of these molecules, $H O X$ transcript antisense intergenic RNA (HOTAIR), has an oncogenic role in the development of human cancer in diverse tissues $[5,7,8]$. HOTAIR is a 2158-nucleotide lncRNA transcribed from the antisense strand of the HOXC gene which is located on chromosome 
12 [9]. HOTAIR represses transcription, but surprisingly, in trans at the $H O X D$ gene cluster on chromosome $2[8,10]$. The IncRNA has been proposed to function as a molecular scaffold for the assembly of polycomb repressive complex 2 (PRC2) and lysine specific demethylase 1/ REST corepressor 1/RE1-silencing transcription factor (LSD1/CoREST/REST) complex at $5^{\prime}$ and $3^{\prime}$ domains, respectively. Histone $\mathrm{H} 3 \mathrm{~K} 27$ methylation and $\mathrm{H} 3 \mathrm{~K} 4$ demethylation activities are thus effectively localized [11], which ultimately results in efficient chromosome condensation and transcriptional repression of targeted genes [7].

Numerous studies have demonstrated that overexpression of HOTAIR occurs in many cancers. It is furthermore associated with poor prognosis, and in experimental models, it promotes tumor progression, invasion, and metastasis [12-16]. Finally, HOTAIR single nucleotide polymorphisms (SNPs) have been investigated as potential cancer susceptibility loci and linked to increased risk for human cancers, such as breast [17-19], esophageal squamous cell carcinoma [20], gastric [21-24], lung [25], and colorectal cancers [26]. However, the results remain controversial possibly due to the fact that independent studies are underpowered and biased, especially for small cohorts. Here, a metaanalysis of eligible studies conducted before January 20, 2016 was performed in order to obtain more precise and comprehensive insight into the impact of HOTAIR polymorphisms on cancer susceptibility. The results indicated that HOTAIR polymorphisms are associated with increased cancer risk, but mainly in stratified analysis based on ethnicity and cancer type.

\section{RESULTS}

\section{Study characteristics}

Our database search yielded 10 studies with a total of 7,772 cases and 9,075 controls that were eligible for our meta-analysis [17-26]. The main features of the eligible studies, which included genotyping method, are listed in Table 1. All were case-control studies and were comprised of individuals of Asian $(n=7)$ and Turkish descent $(n=3)$. In addition, the studies covered diverse tumor types: gastric cancer $(n=4)$ [21-24], breast cancer $(n=3)$ [17-19], colorectal cancer $(n=1)$ [26], lung cancer $(n=1)$ [25], and esophageal squamous cell carcinoma $(n=1)$ [20]. Quality of the included studies was assessed using the Newcastle Ottawa Scale, and all the studies scored a 7 or above (high-quality).

The number of HOTAIR SNPs extracted from all eligible studies was 7. Of these, only 2 SNPs, rs4759314 and $r s 920778$, were reported in 5 or more studies and were thus the focus of the meta-analysis. The genotype distributions of HOTAIR rs4759314 and rs920778 SNPs are shown in Table 2.

\section{HOTAIR polymorphism $r s 920778$ is associated with a general susceptibility cancer}

The first step in the analysis was therefore to determine whether either $r s 4759314$ or $r s 920778$ was associated with a general risk for cancer regardless of tissue origin. Analysis including all individuals from all eligible studies revealed that association between the polymorphism rs 920778 and increased cancer risk was statistically significant in allele contrast ( $\mathrm{T}$ vs $\mathrm{C}, \mathrm{OR}=$ $1.239,95 \% \mathrm{CI}=1.032-1.487, P=0.021)$ and recessive genotype models (TT vs CT $+\mathrm{CC}, \mathrm{OR}=1.614,95 \% \mathrm{CI}$ $=1.082-2.406, P=0.019)$. However, no significant association between the polymorphism $r s 4759314$ and increased susceptibility to cancer in any genotype model was observed. The analysis thus revealed an association between the T allele or the TT genotype for polymorphism rs920778 and increased risk for cancer.

\section{HOTAIR polymorphism $r s 4759314$ is associated with an increased risk for gastric cancer}

To determine whether HOTAIR polymorphisms rs4759314 or $r s 920778$ were associated with risk for a specific cancer type, stratified analyses were performed on the basis of cancer tissue of origin. In this analysis, association of polymorphism rs4759314 with increased gastric cancer susceptibility was statistically significant under allele contrast $(\mathrm{G} v s \mathrm{~A}, \mathrm{OR}=1.262,95 \% \mathrm{CI}=$ $1.073-1.486, P=0.005)$, dominant $(\mathrm{GG}+\mathrm{AG}$ vs AA, $\mathrm{OR}=1.280,95 \% \mathrm{CI}=1.060-1.547, P=0.010)$, and heterozygous (AG vs AA, OR $=1.288,95 \% \mathrm{CI}=1.057$ - 1.570, $P=0.012$ ) models (Figure 1). These results indicated that the $\mathrm{G}$ allele of $r s 4759314$ was linked to increased susceptibility specifically for gastric cancer.

Significant between-study heterogeneity was observed in allele contrast (I-square $=60.3 \%, P=0.027$ ), dominant (I-square $=61.5 \%, P=0.024$ ), and heterozygous models (I-square $=60.4 \%, P=0.027$ ). Therefore, the random-effects model was used to pool the data. In the stratified analysis based on cancer tissue of origin, no significant heterogeneity was found in the gastric cancer group.

\section{HOTAIR polymorphism $r s 920778$ is associated with cancer susceptibility in Asians}

The studies consisted of individuals of Asian and Turkish descent. Therefore associations between the polymorphisms and a general risk for cancer were examined on the basis of ethnicity. All individuals from studies involving rs4759314 were Asian so analysis stratified by ethnicity was performed only for $r s 920778$. A significant association between rs 920778 and cancer susceptibility was observed in Asians under all genetic models with the results as follows: allele contrast ( $\mathrm{T}$ vs $\mathrm{C}$, 
Table 1: Characteristics of studies on association between HOTAIR polymorphisms and cancers

\begin{tabular}{|c|c|c|c|c|c|c|c|c|c|}
\hline Author & Year & Ethnicity & Cases & Controls & $\begin{array}{l}\text { Source of } \\
\text { Controls }\end{array}$ & Cancers & $\begin{array}{l}\text { Single Nucleotide } \\
\text { Polymorphisms }\end{array}$ & $\begin{array}{c}\text { Genotyping } \\
\text { Method }\end{array}$ & $\begin{array}{c}\text { Quality } \\
\text { Score }\end{array}$ \\
\hline Gong & 2016 & Chinese & 498 & 213 & $\mathrm{HB}$ & Lung cancer & $\begin{array}{c}r s 4759314, \\
r s 7958904, \\
r s 1899663\end{array}$ & $\begin{array}{c}\text { MALDI- } \\
\text { TOF mass } \\
\text { spectrometry }\end{array}$ & 7 \\
\hline Yan & 2015 & Chinese & 502 & 504 & PB & Breast cancer & $\begin{array}{c}r s 1899663, \\
r s 4759314, \\
r s 920778\end{array}$ & $\begin{array}{l}\text { PCR-RFLP, } \\
\text { CRS-RFLP }\end{array}$ & 8 \\
\hline Xue & 2015 & Chinese & 1734 & 1855 & HB & $\begin{array}{l}\text { Colorectal } \\
\text { cancer }\end{array}$ & $\begin{array}{c}r s 4759314 \\
r s 7958904 \\
r s 874945\end{array}$ & TaqMan & 7 \\
\hline Pan & 2015 & Chinese & 800 & 1600 & $\mathrm{HB}$ & Gastric cancer & $\begin{array}{c}\text { rs920778, } \\
\text { rs1899663, } \\
r s 4759314\end{array}$ & PCR-RFLP & 7 \\
\hline Guo & 2015 & Chinese & 515 & 654 & $\mathrm{HB}$ & $\begin{array}{c}\text { Gastric cardia } \\
\text { adenocarcinoma }\end{array}$ & $\begin{array}{c}r s 12826786, \\
r s 4759314, \\
r s 10783618\end{array}$ & PCR-RFLP & 7 \\
\hline $\mathrm{Du}$ & 2015 & Chinese & 1275 & 1646 & $\mathrm{HB}$ & Gastric cancer & $\begin{array}{c}r s 4759314, \\
r s 7958904, \\
r s 874945\end{array}$ & TaqMan & 8 \\
\hline Bayram & 2015 & Turkish & 123 & 122 & HB & Breast cancer & $r s 12826786$ & TaqMan & 7 \\
\hline Bayram & 2015 & Turkish & 104 & 209 & $\mathrm{HB}$ & Gastric cancer & rs920778 & TaqMan & 7 \\
\hline Bayram & 2015 & Turkish & 123 & 122 & HB & Breast cancer & rs920778 & TaqMan & 7 \\
\hline Zhang & 2014 & Chinese & 2098 & 2150 & HB & $\begin{array}{l}\text { Esophageal } \\
\text { squamous cell } \\
\text { carcinoma }\end{array}$ & $\begin{array}{c}r s 920778, \\
r s 1899663, \\
r s 4759314\end{array}$ & PCR-RFLP & 8 \\
\hline
\end{tabular}

PB, population-based; HB, hospital-based; PCR-RFLP, polymerase chain reaction-restriction fragment length polymorphism; CRS-RFLP, created-restriction-site PCR-RFLP.

$\mathrm{OR}=1.456,95 \% \mathrm{CI}=1.349-1.571, P<0.001)$; dominant model $(\mathrm{TT}+\mathrm{CT}$ vs $\mathrm{CC}, \mathrm{OR}=1.462,95 \% \mathrm{CI}=1.325-$ 1.613, $P<0.001)$; recessive model (TT vs $\mathrm{CT}+\mathrm{CC}, \mathrm{OR}=$ $2.128,95 \% \mathrm{CI}=1.417-3.197, P<0.001)$; homozygous model (TT vs CC, OR $=2.764,95 \% \mathrm{CI}=2.221-3.440$, $P<0.001)$; and heterozygous model (CT vs $\mathrm{CC}, \mathrm{OR}=$ $1.323,95 \% \mathrm{CI}=1.194-1.466, P<0.001)$ (Figure 2 ). The $\mathrm{T}$ allele or the TT genotype $r s 920778$ thus emerged as a potential genetic marker for increased cancer susceptibility especially in Asians.

Significant between-study heterogeneity was observed in all genetic models; therefore, analysis using the random-effects model was conducted on data stratified by ethnicity. Under these conditions, statistically significant heterogeneity was no longer observed within Asian or Turkish groups.

\section{Sensitivity analysis}

The stability of the results of the meta-analysis was assessed in sensitivity analysis, where the effect of each study on the pooled OR was examined by repeating the meta-analysis after sequentially omitting each study. Through the deletion of only a single study which was based on a Turkish population [18], an association of the polymorphism rs 920778 with cancer risk emerged under dominant, homozygous, and heterozygous models. The $P$-values were not highly significant, however (data not shown). Therefore, the results of this metaanalysis including all eligible studies were generally and statistically stable.

\section{Publication bias}

Publication bias for each polymorphism in each model was evaluated using both the Begg's and Egger's tests. No obvious asymmetry was observed in any of the Begg's funnel plots indicating that publication bias was generally not a factor influencing the results (Figure 3). All $P$-values from the Egger's test and the Begg's test are listed in Table 3 . The values are consistent with the absence of significant publication bias in the analysis of 
Table 2: Genotype distributions of HOTAIR polymorphisms rs4759314 and rs920778

\begin{tabular}{|c|c|c|c|c|c|c|c|}
\hline$r s 4759314$ & \multicolumn{2}{|c|}{ AA genotype } & \multicolumn{2}{|c|}{ AG genotype } & \multicolumn{2}{|c|}{ GG genotype } & \multirow{2}{*}{$\begin{array}{l}P \text { for } \mathrm{HWE} \\
\text { in controls }\end{array}$} \\
\hline Study & Cases & Controls & Cases & Controls & Cases & Controls & \\
\hline Yan (2015) & 451 & 448 & 50 & 54 & 1 & 2 & 0.78 \\
\hline Xue (2015) & 1528 & 1608 & 200 & 236 & 5 & 11 & 0.47 \\
\hline Pan (2015) & 451 & 914 & 48 & 83 & 1 & 3 & 0.45 \\
\hline Guo (2015) & 461 & 589 & 53 & 64 & 1 & 1 & 0.59 \\
\hline Du (2015) & 1083 & 1464 & 186 & 172 & 6 & 8 & 0.23 \\
\hline Zhang (2014) & 917 & 910 & 81 & 89 & 2 & 1 & 0.44 \\
\hline$r s 920778$ & \multicolumn{2}{|c|}{ CC genotype } & \multicolumn{2}{|c|}{ CT genotype } & \multicolumn{2}{|c|}{ TT genotype } & $P$ for $\mathrm{HWE}$ \\
\hline Study & Cases & Controls & Cases & Controls & Cases & Controls & in controls \\
\hline Yan (2015) & 12 & 18 & 151 & 190 & 339 & 296 & 0.06 \\
\hline Pan (2015) & 420 & 980 & 321 & 575 & 59 & 45 & $<0.01$ \\
\hline Bayram (2015-May) & 20 & 38 & 52 & 105 & 32 & 66 & 0.74 \\
\hline Bayram (2015-Jan) & 31 & 15 & 52 & 66 & 40 & 41 & 0.14 \\
\hline Zhang (2014) & 1091 & 1323 & 826 & 749 & 181 & 78 & 0.03 \\
\hline
\end{tabular}

HWE, Hardy-Weinberg equilibrium.

cancer risk and polymorphisms in most genotype models except in the case of polymorphism rs 920778 . For this polymorphism, publication bias was apparent in the allele contrast model. However, analysis with the trim and fill method demonstrated that the results of our study did not significantly change even after adjusting for the publication bias.

\section{DISCUSSION}

The overexpression of HOTAIR in various cancer types has led to its examination as a candidate molecule for the diagnosis and treatment of the disease. Here, HOTAIR was investigated for potential links to cancer susceptibility. We performed a metaanalysis using data from available cases $(n=7,772)$ and controls $(n=9,075)$ in the literature to date to provide further evidence that HOTAIR polymorphisms rs4759314 and rs920778 are associated with cancer risk. Only polymorphism $r s 920778$ was found to be generally associated with increased risk for cancer, but statistically significant associations for both polymorphisms were revealed when data were stratified based on ethnicity and cancer type.

The results of our analysis indicated that the $\mathrm{T}$ allele or TT genotype of polymorphism is a potential genetic marker for cancer susceptibility, especially in Asians, and that the $G$ allele in polymorphism rs4759314 might be related to susceptibility for gastric cancer. A possible mechanism underlying the association of these alleles to increased susceptibility is through the regulation of the expression of HOTAIR itself. Polymorphism rs4759314 resides in an intronic promoter region, which was found to influence the activity of this promoter and expression of $\mathrm{HOXC11}$ gene [23]. For polymorphism $r_{s} 4759314$, increased HOTAIR expression originated from the $\mathrm{G}$ (relative to the A) allele in luciferase reporter assays. A similar finding was made for polymorphism $r s 920778$, which resides in a novel HOTAIR intronic enhancer. In this case, increased HOTAIR expression was associated with $\mathrm{T}$ allele carriers [20,22]. For both polymorphisms, the results of the functional assay correlated with the results of our meta-analysis; higher risk alleles drove increased expression of HOTAIR.

A sufficient number of cases and controls were pooled from different studies and provided a more accurate estimation of the associations between the HOTAIR polymorphisms and cancer risk as compared to individual studies. However, some limitations of this meta-analysis exist. First, although the analysis was performed with strict criteria for study inclusion and precise data extraction, significant between-study heterogeneity existed in some comparisons. However, after analysis stratified by cancer type or ethnicity, heterogeneity between the subgroups was significantly reduced. Second, our analysis was limited to individuals of Asian and Turkish descent so that it remains unclear as to whether these results can be generalized to other populations. 
A

Study

$\%$

ID

OR $(95 \% \mathrm{Cl})$

Weight

Breast cancer

Yan (2015)

Subtotal (l-squared $=. \%, p=$.)

Colorectal cancer

Xue (2015)

Subtotal (l-squared $=\%, \mathrm{p}=$.)

Gastric cancer

Pan (2015)

Guo (2015)

Du (2015)

Subtotal (l-squared $=1.9 \%, p=0.361$ )

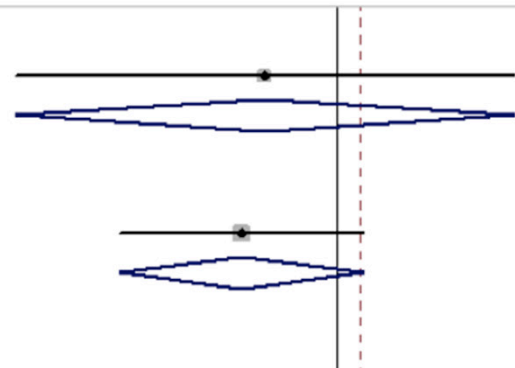

$0.89(0.61,1.31) \quad 12.78$

$0.89(0.61,1.31) \quad 12.78$

Esophageal squamous cell carcinoma

Zhang (2014)

Subtotal $(1-$ squared $=\%, p=$.)

Overall (l-squared $=60.3 \%, p=0.027$ )

NOTE: Weights are from random effects analysis

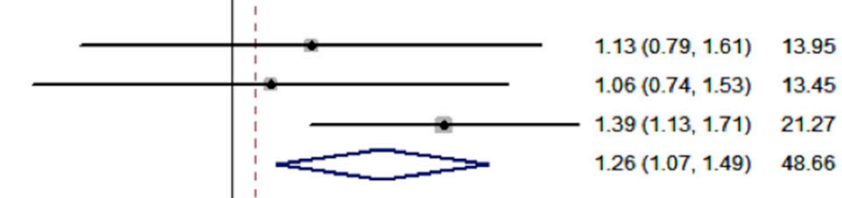

B

Study

ID

Breast cancer

Yan (2015)

Subtotal (l-squared $=\%, p=$.)

Colorectal cancer

Xue (2015)

Subtotal (l-squared $=\%, p=$ )

Gastric cancer

Pan (2015)

Guo (2015)

Du (2015)

Subtotal (l-squared $=15.0 \%, p=0.308$ )

Esophageal squamous cell carcinoma

Zhang (2014)

Subtotal (I-squared $=. \%, p=$,

Overall ( $I$-squared $=61.5 \%, p=0.024)$

NOTE: Weights are from random effects analysis

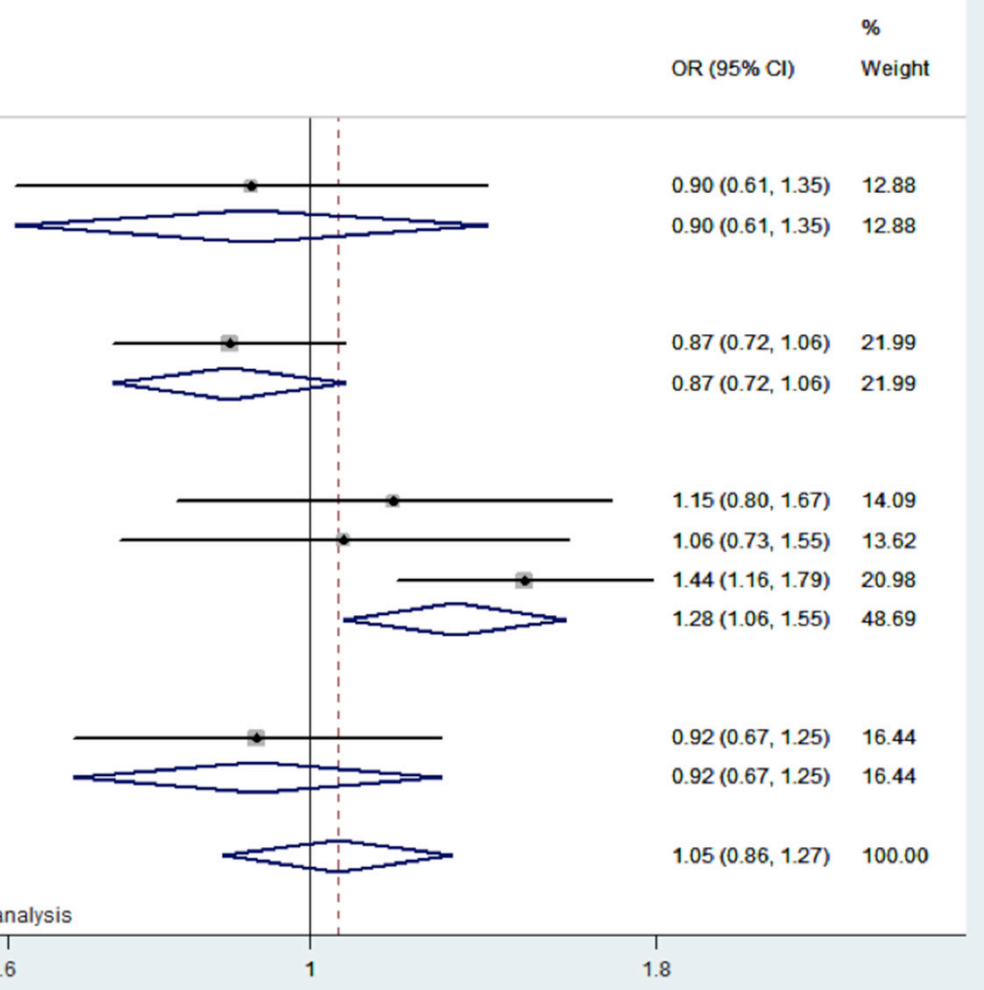

$0.86(0.71,1.04) \quad 22.28$

$0.86(0.71,1.04) \quad 22.28$

$0.93(0.69,1.26) \quad 16.27$

$0.93(0.69,1.26) \quad 16.27$

$1.04(0.86,1.25) \quad 100.00$

1.71

Figure 1: Forest plot of cancer risk in different cancer types associated with HOTAIR polymorphism rs4759314. Models represented in A. allele contrast and $\mathbf{B}$. dominant. 
A

Study

$\%$

ID

OR $(95 \% \mathrm{Cl}) \quad$ Weight

\section{Asian}

Yan (2015)

Pan (2015)

Zhang (2014)

Subtotal $(1-$ squared $=0.0 \%, p=0.803)$

Turkish

Bayram (2015-May)

Bayram (2015-Jan)

Subtotal (l-squared $=0.0 \%, p=0.321$ )

Overall (I-squared $=77.5 \%, p=0.001)$

NOTE: Weights are from random effects analysis

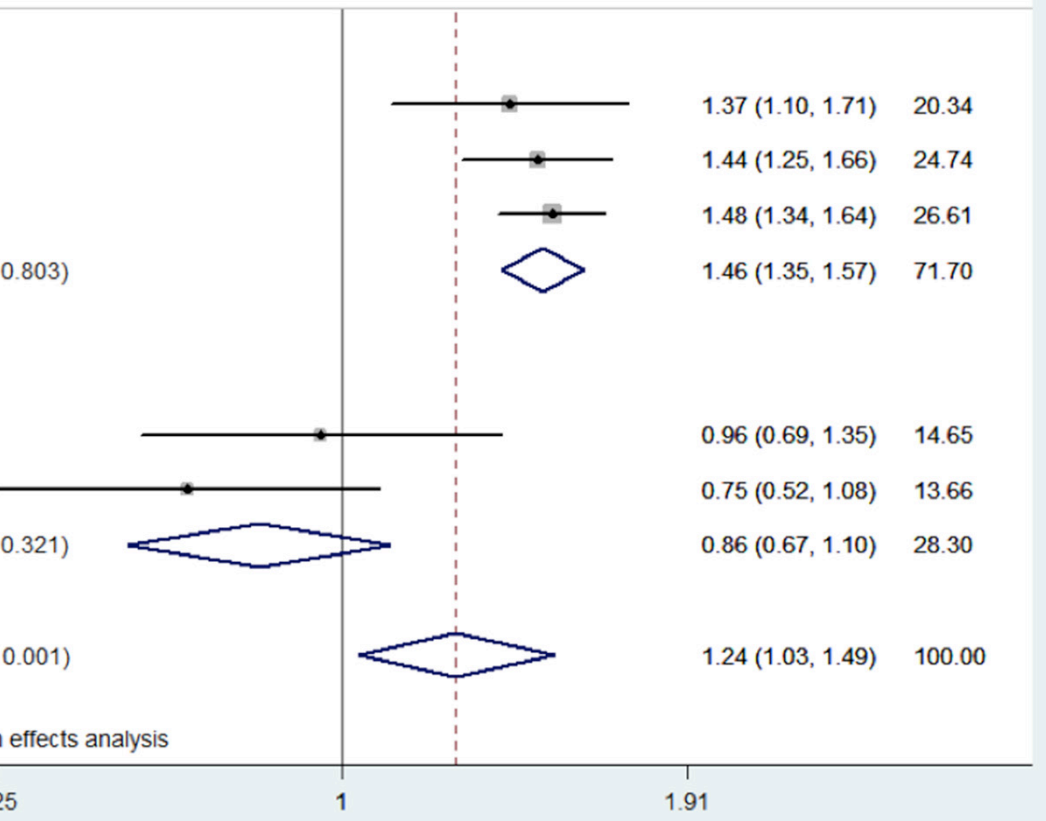

B

Study

ID

\section{Asian}

Yan (2015)

Pan (2015)

Zhang (2014)

Subtotal $(I-$ squared $=81.5 \%, p=0.004)$

Turkish

Bayram (2015-May)

Bayram (2015-Jan)

Subtotal (l-squared $=0.0 \%, p=0.976)$

Overall $(\mathrm{l}$-squared $=83.2 \%, p=0.000)$

NOTE: Weights are from random effects analysis

244

\section{OR $(95 \% \mathrm{Cl}) \quad$ Weight}

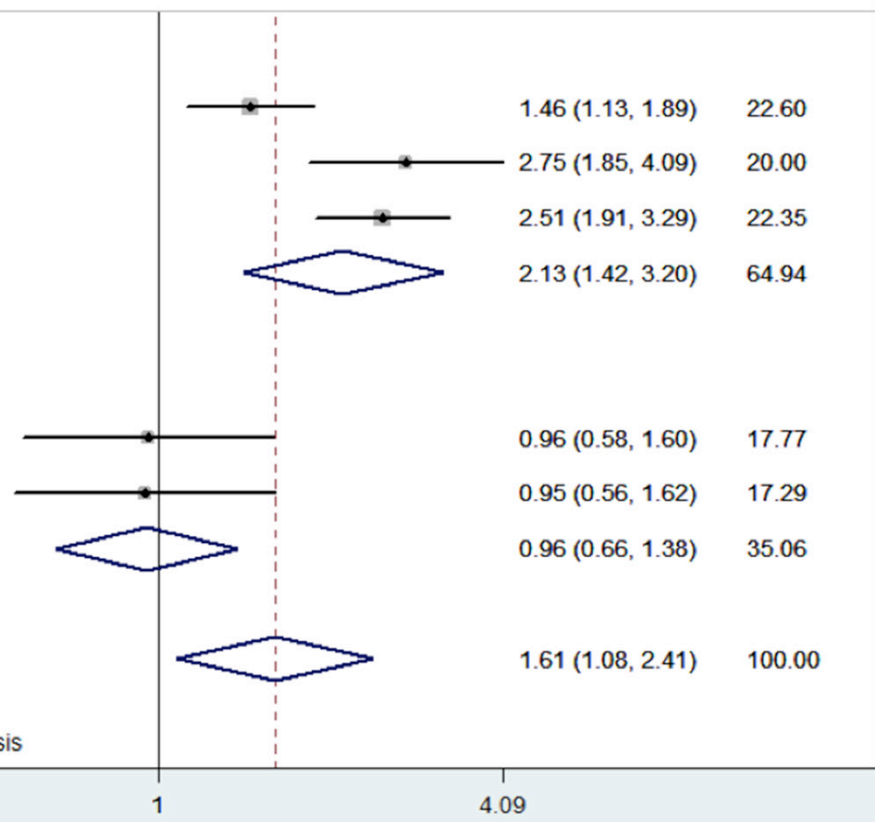

Figure 2: Forest plot of cancer risk in different ethnicities associated with HOTAIR polymorphism rs920778. Models represented A. allele contrast and $\mathbf{B}$. recessive. 
In conclusion, our results indicate that HOTAIR polymorphism $r_{s} 920778$ is more generally associated with cancer risk, particularly in Asians, whereas polymorphism rs 4759314 may be a risk factor for gastric cancer. However, these polymorphisms require further evaluation in future well-designed studies as potential genetic susceptibility loci in different cancers as well as ethnic populations.

\section{MATERIALS AND METHODS}

\section{Search strategy}

PubMed, Embase, and Web of Science databases were searched for studies reporting association of HOTAIR polymorphisms with cancer risk up to the date of January 20, 2016. The literature search was performed

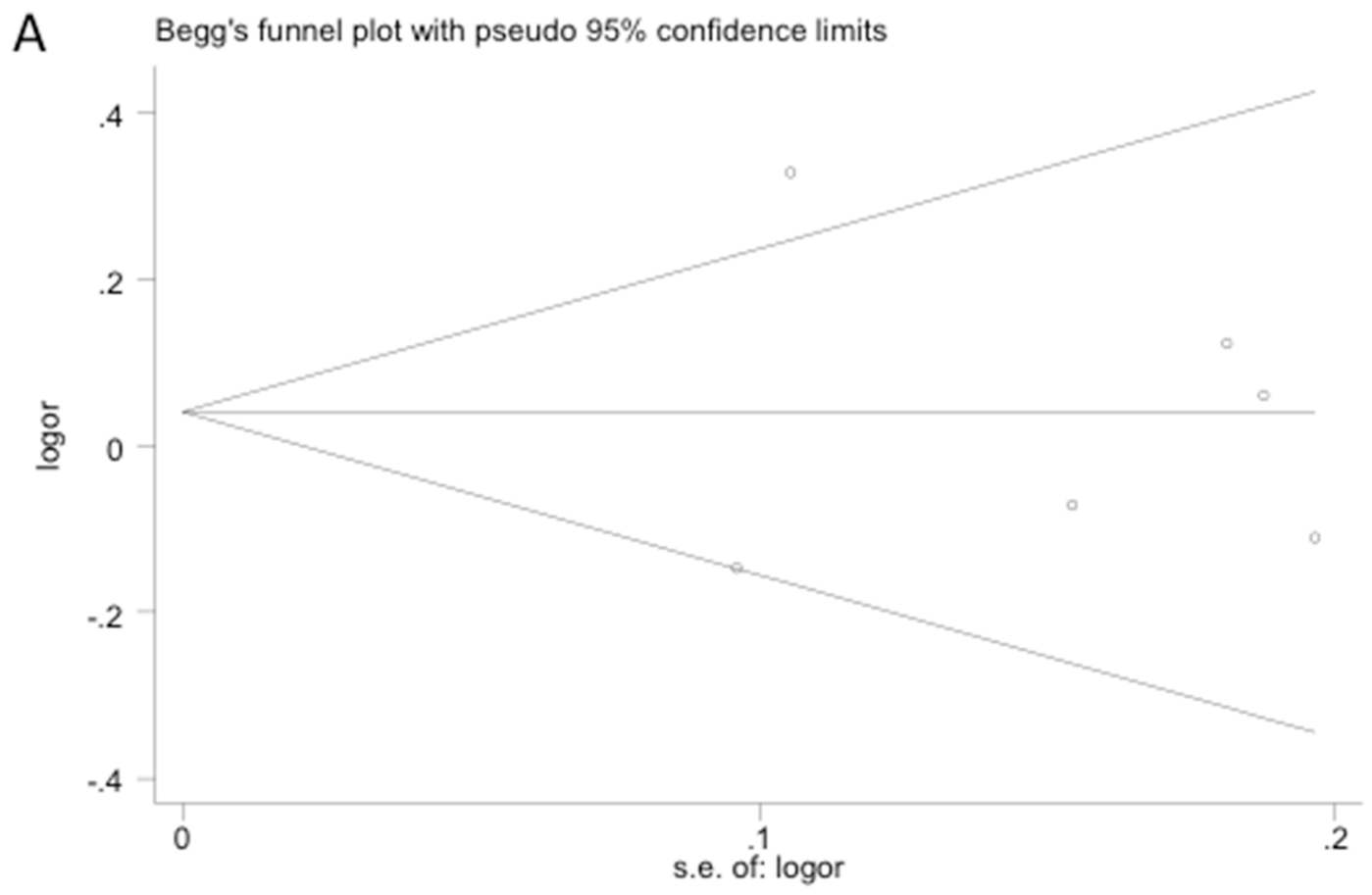

B Begg's funnel plot with pseudo $95 \%$ confidence limits

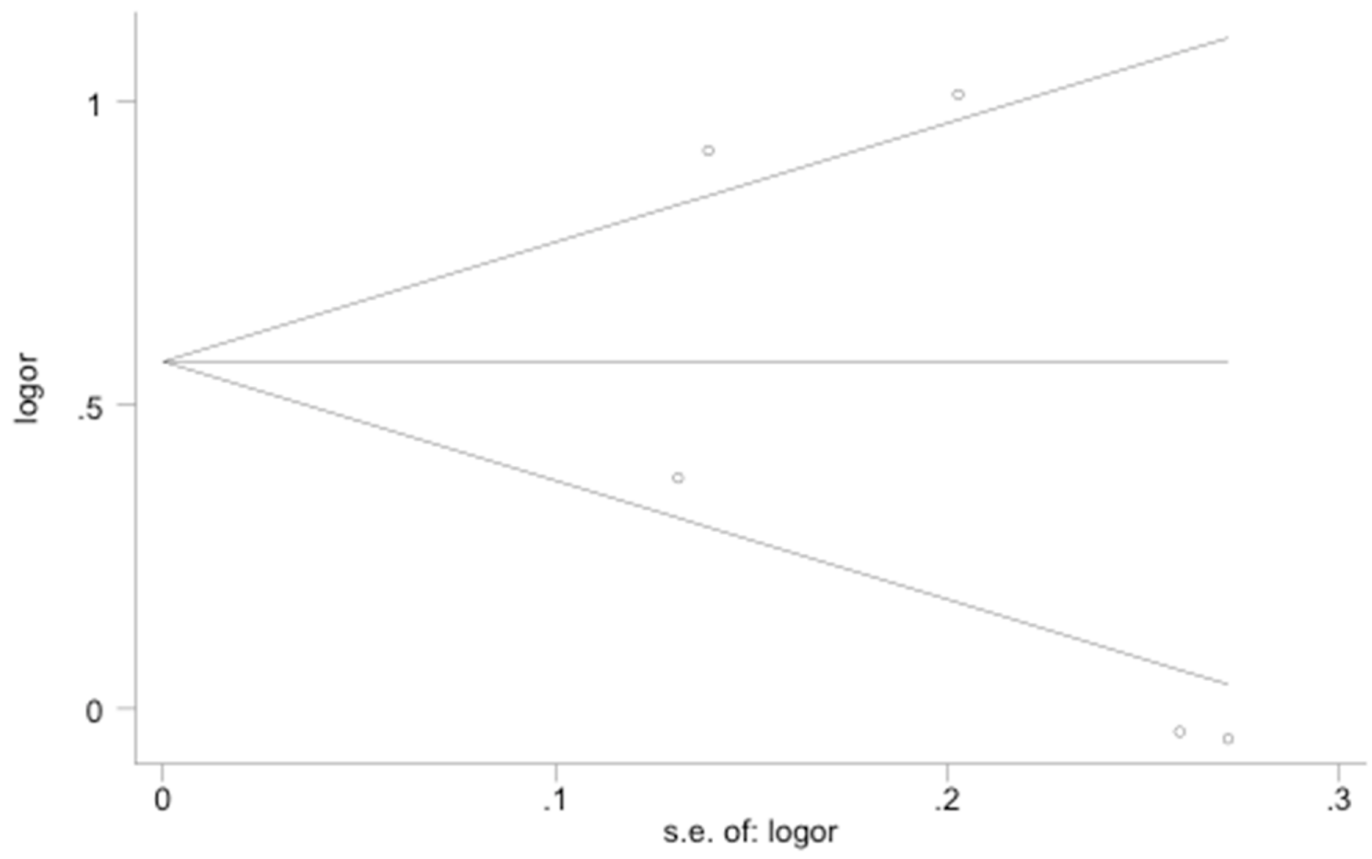

Figure 3: Publication bias tested by Begg's funnel plot. Polymorphisms represented in A. $r s 4759314$ and B. $r s 920778$. 
Table 3: ORs and 95\% CI for cancers and the HOTAIR polymorphisms under different genetic models

\begin{tabular}{|c|c|c|c|c|c|c|c|c|}
\hline Genetic models & $\mathbf{n}$ & OR [95\% CI] & $P(\mathrm{OR})$ & $\begin{array}{c}\text { Model } \\
\text { (method) }\end{array}$ & $\begin{array}{c}\text { I-square } \\
(\%)\end{array}$ & $P(\mathbf{H})$ & $P$ (Begg) & $P$ (Egger) \\
\hline
\end{tabular}

$r s 4759314$

Allele contrast ( $\mathrm{G}$ vs A)

$\begin{array}{lllllllll}\text { All } & 6 & 1.037[0.863-1.246] & 0.700 & \text { R (D-L) } & 60.3 & 0.027 & 1.000 & 0.903 \\ \text { Gastric cancer } & 3 & 1.262[1.073-1.486] & 0.005 & \text { R (D-L) } & 1.9 & 0.361 & - & -\end{array}$

Dominant model (GG+AG vs AA)

$\begin{array}{lllllllll}\text { All } & 6 & 1.049[0.864-1.275] & 0.629 & \text { R (D-L) } & 61.5 & 0.024 & 1.000 & 0.868 \\ \text { Gastric cancer } & 3 & 1.280[1.060-1.547] & 0.010 & \text { R (D-L) } & 15.0 & 0.308 & - & -\end{array}$

Recessive model (GG vs AG+AA)

$\begin{array}{lllllllll}\text { All } & 6 & 0.739[0.395-1.381] & 0.343 & \text { F (M-H) } & 0.0 & 0.880 & 0.707 & 0.495 \\ \text { Gastric cancer } & 3 & 0.933[0.378-2.300] & 0.880 & \text { F (M-H) } & 0.0 & 0.934 & - & -\end{array}$

Homozygous model (GG vs AA)

$\begin{array}{lllllllll}\text { All } & 6 & 0.747[0.400-1.396] & 0.361 & \text { F }(\mathrm{M}-\mathrm{H}) & 0.0 & 0.863 & 0.707 & 0.532 \\ \text { Gastric cancer } & 3 & 0.968[0.393-2.387] & 0.944 & \text { F (M-H) } & 0.0 & 0.931 & - & -\end{array}$

Heterozygous model (AG vs AA)

$\begin{array}{lllllllll}\text { All } & 6 & 1.059[0.872-1.286] & 0.566 & \text { R (D-L) } & 60.4 & 0.027 & 1.000 & 0.833 \\ \text { Gastric cancer } & 3 & 1.288[1.057-1.570] & 0.012 & \text { R (D-L) } & 19.1 & 0.291 & - & -\end{array}$

rs920778

Allele contrast ( $\mathrm{T} v s \mathrm{C}$ )

$\begin{array}{lcccccccc}\text { All } & 5 & 1.239[1.032-1.487] & 0.021 & \text { R (D-L) } & 77.5 & 0.001 & 0.027 & 0.030 \\ \text { Asian } & 3 & 1.456[1.349-1.571] & <0.001 & \text { R (D-L) } & 0.0 & 0.803 & - & -\end{array}$

Dominant model (TT+CT vs CC)

$\begin{array}{lcccccccc}\text { All } & 5 & 1.199[0.916-1.570] & 0.186 & \text { R (D-L) } & 73.2 & 0.005 & 0.221 & 0.170 \\ \text { Asian } & 3 & 1.462[1.325-1.613] & <0.001 & \text { R (D-L) } & 0.0 & 0.953 & - & -\end{array}$

Recessive model (TT vs CT+CC)

$\begin{array}{lllllllll}\text { All } & 5 & 1.614[1.082-2.406] & 0.019 & \text { R (D-L) } & 83.2 & <0.001 & 0.462 & 0.442 \\ \text { Asian } & 3 & 2.128[1.417-3.197] & <0.001 & \text { R (D-L) } & 81.5 & 0.004 & - & -\end{array}$

Homozygous model (TT vs CC)

$\begin{array}{lcccccccc}\text { All } & 5 & 1.549[0.843-2.846] & 0.159 & \text { R (D-L) } & 85.8 & <0.001 & 0.086 & 0.078 \\ \text { Asian } & 3 & 2.764[2.221-3.440] & <0.001 & \text { R (D-L) } & 0.0 & 0.403 & - & -\end{array}$

Heterozygous model (CT vs CC)

\begin{tabular}{lcccccccc} 
All & 5 & $1.115[0.862-1.441]$ & 0.407 & $\mathrm{R}(\mathrm{D}-\mathrm{L})$ & 67.8 & 0.014 & 0.221 & 0.143 \\
Asian & 3 & $1.323[1.194-1.466]$ & $<0.001$ & $\mathrm{R}(\mathrm{D}-\mathrm{L})$ & 0.0 & 0.938 & - & - \\
\hline
\end{tabular}

OR, Odds ratio; CI, confidence intervals; $P(\mathrm{H}), P$ for heterogeneity; $\mathrm{n}$, number of included studies; $\mathrm{F}$, fixed-effect model; R, random-effects model; M-H, Mantel-Haenszel method; D-L, DerSimonian-Laird method.

using free-text words combined with Medical Subject Headings (MeSH), such as "Neoplasms", "HOTAIR long untranslated RNA, human" and "Polymorphism, Single Nucleotide". Gene-specific terms (HOX transcript antisense intergenic RNA or HOTAIR) were combined with polymorphism-specific terms (polymorphism or polymorphisms or variation or variations or variant or variants or mutation or mutations or genotype or 
genotypes) and disease-specific terms (cancer or cancers or tumor or tumors or neoplasm or neoplasms) to retrieve eligible studies. References cited in retrieved articles were also reviewed to identify additional potentially relevant studies.

\section{Inclusion and exclusion criteria}

Inclusion criteria for studies were the following: (1) case-control or cohort study design; (2) evaluating associations between HOTAIR polymorphisms and all types of cancer; (3) providing sufficient data for SNP allele and genotype frequencies; (4) published in English; and (5) performed on humans. Exclusion criteria were the following: (1) reviews and comments; (2) performed on animals, and (3) duplication of a previous publication.

\section{Data extraction}

Two investigators (QQ and JW) independently extracted the following data from each study: first author's surname, publication year, ethnicity, cancer types, numbers of cases and controls, and genotype distributions of cases and controls. Different ethnicity descents were categorized as Asian or Turkish. Study design was stratified into population-based and hospital-based studies. The results were compared and disagreement was resolved by discussion with a third reviewer $(\mathrm{BH})$ until consensus was reached.

\section{Quality assessment}

The Newcastle-Ottawa Scale and Agency for Healthcare Research and Quality (http:/www.ohri.ca/ programs/clinical_epidemiology/oxford.asp; maximum score $=9$ points) was used to evaluate the methodological quality, which scored studies based on the selection of patients, the comparability of the groups, and the quality of the sampling process. A study awarded a score of $0-3$, 4 - 6, or 7 - 9 was considered as a low-, moderate-, or high-quality study, respectively.

\section{Statistical analysis}

To obtain a more comprehensive assessment of associations between HOTAIR polymorphisms and cancer susceptibility, five different comparison models were used: allele contrast, dominant, recessive, homozygous and heterozygous. Odds ratio (OR) with 95\% confidence intervals (CI) was used to estimate the strength of associations, and the significance of ORs was determined with the $\mathrm{Z}$ test. Heterogeneity among the included studies was assessed by Chi square-based $\mathrm{Q}$ statistic. A randomeffects (DerSimonian-Laird method) or fixed-effect (Mantel-Haenszel method) model was used to calculate pooled effect estimates in the presence $(P<0.05)$ or absence $(P>0.05)$ of heterogeneity. The method of subgroup analysis according to the ethnicity or cancer type of the participants was also applied to gain more precise results. Sensitivity analysis was conducted by sequentially excluding each study. The Begg's test and the Egger's test were performed to analyze for the presence of publication bias. If any possible bias was observed, the trim and fill method was used to identify and adjust for those studies. Data analyses were carried out using Stata software, version 11.0 (Stata Corporation; College Station, TX, USA). $P$ values $<0.05$ were considered statistically significant.

\section{ACKNOWLEDGMENTS}

This work was supported by Natural Science Foundation of China Grant (grants 81502164, 81402060 and 81572487), the Shandong Provincial Natural Science Foundation Grant (grants BS2015YY004 and BS2014YY033), the Special foundation for Taishan Scholars (grants ts20110814 and tshw201502056), the Fundamental Research Funds of Shandong University, the Department of Science \& Technology of Shandong Province (grant 2015GGE27101 and 2015ZDXX0801A01), the University of Bergen, Helse Bergen, Norway and the Norwegian Centre for International Cooperation in Education (SIU)(UTF-2014/10047).

\section{CONFLICTS OF INTEREST}

The authors declare no conflicts of interest.

\section{REFERENCES}

1. Ponting CP, Oliver PL and Reik W. Evolution and functions of long noncoding RNAs. Cell. 2009; 136:629-641.

2. Mercer TR, Dinger ME and Mattick JS. Long non-coding RNAs: insights into functions. Nat Rev Genet. 2009; 10:155-159.

3. Qiu MT, Hu JW, Yin R and Xu L. Long noncoding RNA: an emerging paradigm of cancer research. Tumour Biol. 2013; 34:613-620.

4. Yang G, Lu X and Yuan L. LncRNA: a link between RNA and cancer. Biochim Biophys Acta. 2014; 1839:1097-1109.

5. Gibb EA, Brown CJ and Lam WL. The functional role of long non-coding RNA in human carcinomas. Mol Cancer. 2011; 10:38.

6. Gutschner T and Diederichs S. The hallmarks of cancer: a long non-coding RNA point of view. RNA Biol. 2012; 9:703-719.

7. Bhan A and Mandal SS. LncRNA HOTAIR: A master regulator of chromatin dynamics and cancer. Biochim Biophys Acta. 2015; 1856:151-164. 
8. Wu Y, Zhang L, Wang Y, Li H, Ren X, Wei F, Yu W, Wang $\mathrm{X}$, Zhang L, Yu J and Hao X. Long noncoding RNA HOTAIR involvement in cancer. Tumour Biol. 2014; 35:9531-9538.

9. Woo CJ and Kingston RE. HOTAIR lifts noncoding RNAs to new levels. Cell. 2007; 129:1257-1259.

10. Zhang S, Chen S, Yang G, Gu F, Li M, Zhong B, Hu J, Hoffman A and Chen M. Long noncoding RNA HOTAIR as an independent prognostic marker in cancer: a metaanalysis. PLoS One. 2014; 9:e105538.

11. Tsai MC, Manor O, Wan Y, Mosammaparast N, Wang JK, Lan F, Shi Y, Segal E and Chang HY. Long noncoding RNA as modular scaffold of histone modification complexes. Science. 2010; 329:689-693.

12. Endo H, Shiroki T, Nakagawa T, Yokoyama M, Tamai K, Yamanami H, Fujiya T, Sato I, Yamaguchi K, Tanaka N, Iijima K, Shimosegawa T, Sugamura K and Satoh K. Enhanced expression of long non-coding RNA HOTAIR is associated with the development of gastric cancer. PLoS One. 2013; 8:e77070.

13. Kim K, Jutooru I, Chadalapaka G, Johnson G, Frank J, Burghardt R, Kim S and Safe S. HOTAIR is a negative prognostic factor and exhibits pro-oncogenic activity in pancreatic cancer. Oncogene. 2013; 32:1616-1625.

14. Liu XH, Liu ZL, Sun M, Liu J, Wang ZX and De W. The long non-coding RNA HOTAIR indicates a poor prognosis and promotes metastasis in non-small cell lung cancer. BMC Cancer. 2013; 13:464.

15. Su X, Malouf GG, Chen Y, Zhang J, Yao H, Valero V, Weinstein JN, Spano JP, Meric-Bernstam F, Khayat D and Esteva FJ. Comprehensive analysis of long non-coding RNAs in human breast cancer clinical subtypes. Oncotarget. 2014; 5:9864-9876. doi: 10.18632/oncotarget.2454.

16. Zhang JX, Han L, Bao ZS, Wang YY, Chen LY, Yan W, Yu SZ, Pu PY, Liu N, You YP, Jiang T and Kang CS. HOTAIR, a cell cycle-associated long noncoding RNA and a strong predictor of survival, is preferentially expressed in classical and mesenchymal glioma. Neuro Oncol. 2013; 15:1595-1603.

17. Yan R, Cao J, Song C, Chen Y, Wu Z, Wang K and Dai L. Polymorphisms in lncRNA HOTAIR and susceptibility to breast cancer in a Chinese population. Cancer Epidemiol. 2015; 39:978-985.
18. Bayram S, Sumbul AT, Batmaci CY and Genc A. Effect of HOTAIR rs920778 polymorphism on breast cancer susceptibility and clinicopathologic features in a Turkish population. Tumour Biol. 2015; 36:3863-3870.

19. Bayram S, Sumbul AT and Dadas E. A functional HOTAIR rs12826786 C $>\mathrm{T}$ polymorphism is associated with breast cancer susceptibility and poor clinicopathological characteristics in a Turkish population: a hospital-based case-control study. Tumour Biol. 2016; 37:5577-5584.

20. Zhang X, Zhou L, Fu G, Sun F, Shi J, Wei J, Lu C, Zhou C, Yuan Q and Yang M. The identification of an ESCC susceptibility SNP rs920778 that regulates the expression of lncRNA HOTAIR via a novel intronic enhancer. Carcinogenesis. 2014; 35:2062-2067.

21. Bayram S, Ulger Y, Sumbul AT, Kaya BY, Rencuzogullari A, Genc A, Sevgiler Y, Bozkurt O and Rencuzogullari E. A functional HOTAIR rs920778 polymorphism does not contributes to gastric cancer in a Turkish population: a casecontrol study. Fam Cancer. 2015; 14:561-567.

22. Pan W, Liu L, Wei J, Ge Y, Zhang J, Chen H, Zhou L, Yuan Q, Zhou C and Yang M. A functional lncRNA HOTAIR genetic variant contributes to gastric cancer susceptibility. Mol Carcinog. 2015.

23. Du M, Wang W, Jin H, Wang Q, Ge Y, Lu J, Ma G, Chu $\mathrm{H}$, Tong N, Zhu H, Wang M, Qiang F and Zhang Z. The association analysis of lncRNA HOTAIR genetic variants and gastric cancer risk in a Chinese population. Oncotarget. 2015; 6:31255-31262. doi: 10.18632/oncotarget.5158.

24. Guo W, Dong Z, Bai Y, Guo Y, Shen S, Kuang G and Xu J. Associations between polymorphisms of HOTAIR and risk of gastric cardia adenocarcinoma in a population of north China. Tumour Biol. 2015; 36:2845-2854.

25. Gong WJ, Yin JY, Li XP, Fang C, Xiao D, Zhang W, Zhou $\mathrm{HH}$, Li X and Liu ZQ. Association of well-characterized lung cancer lncRNA polymorphisms with lung cancer susceptibility and platinum-based chemotherapy response. Tumour Biol. 2016.

26. Xue Y, Gu D, Ma G, Zhu L, Hua Q, Chu H, Tong N, Chen $J$, Zhang $Z$ and Wang $M$. Genetic variants in lncRNA HOTAIR are associated with risk of colorectal cancer. Mutagenesis. 2015; 30:303-310. 